\title{
Hemolytic uremic syndrome with multiple organ involvement secondary to complement factor $\mathrm{H}$ p.Arg1215X mutation
}

\author{
Osman Yeşilbaş ${ }^{1}$, Esra Şevketoğlu${ }^{1}$, Mey Talip Petmezci ${ }^{1}$, Hasan Serdar Kıhtır ${ }^{1}$, Meryem \\ Benzer ${ }^{2}$, Afig Berdeli ${ }^{3}$ \\ Departments of ${ }^{1}$ Pediatric Critical Care, ${ }^{2}$ Nephrology, Bakirkoy Dr. Sadi Konuk Research and Training Hospital, Istanbul; \\ ${ }^{3}$ Department of Pediatrics, Molecular Medicine Laboratory and Stem Cell Department of Health Science Institute, Ege \\ University Faculty of Medicine Izmir, Turkey. E-mail: drosmanyesilbas@gmail.com \\ Received: 5th April 2017, Accepted: 19th May 2017
}

SUMMARY: Yeşilbaş O, Şevketoğlu E, Petmezci MT, Kıhtır HS, Benzer M, Berdeli A. Hemolytic uremic syndrome with multiple organ involvement secondary to complement factor H p.Arg1215X mutation. Turk J Pediatr 2017; 59: 576-580.

Complement mediated hemolytic uremic syndrome which is caused by excessive activation of the alternative complement system is a thrombotic microangiopathy. The disease frequently occurs as a result of mutations in the genes that regulates complement proteins. Complement factor $\mathrm{H}$ gene has the most common mutations. A nine-month-old male patient was transferred to pediatric intensive care unit with the diagnosis of hemolytic uremic syndrome. Nonsense heterozygous p.Arg1215X mutation in the complement factor $\mathrm{H}$ gene was detected. The patient who had pulmonary, intestinal and hepatic involvement accompanying acute renal failure was successfully treated with therapeutic plasma exchange and eculizumab. Nonsense heterozygous p.Arg $1215 \mathrm{X}$ mutation is extremely rare and can cause severe hemolytic uremic syndrome. As far as we know, our patient is the third case with this mutation in the literature.

Key words: complement factor $H$ gene, hemolytic uremic syndrome, pulmonary edema, therapeutic plasma exchange

Hemolytic uremic syndrome (HUS) is characterized by nonimmune hemolytic anemia, acute renal failure (ARF) and thrombocytopenia. As a result of complement mutations, alternative complement system is activated uncontrollably and can not be sufficiently suppressed in complement mediated HUS (CM-HUS).1,2 Eventually, endothelial damage and secondary to it thrombotic microangiopathy (TMA) occurs in all organ systems of the body, especially in the renal vessels. Complement factor $\mathrm{H}$ $(\mathrm{CFH})$ is the main regulator and the most frequent mutations identified gene of alternative complement system. ${ }^{1-3}$ In this article, we present an extremely rare CM-HUS case with the heterozygous p.Arg $1215 \mathrm{X}$ terminating mutation in the CFH gene. The patient with pulmonary, intestinal and hepatic involvement accompanying ARF was successfully treated with therapeutic plasma exchange (TPE) followed by eculizumab (a human anti-C5 monoclonal antibody).

\section{Case Report}

A nine-month-old male patient was admitted to a hospital with one week history of vomiting, fatigue, and anorexia. Because of anemia, thrombocytopenia, oligo-anuric renal failure, deterioration in kidney function tests, lack of bloody diarrhea before period, and his young age; CM-HUS was considered and periton dialysis was planned to start. With the addition of respiratory failure at follow-up, the patient was referred to our pediatric intensive care unit (PICU).

Physical examination was as follows; poor general condition, lethargic (Glasgow Coma Scale 12), prolonged capillary refill time (4 seconds), tachypneic $(58 / \mathrm{min})$, tachycardic (183/min), and 109/77 mm Hg (MAP $92 \mathrm{~mm}$ $\mathrm{Hg}$ ) blood pressure. He had moderate-diffused edema on his eyelids and body. In order to keep the oxygen saturation above $94 \%$, he was receiving $10 \mathrm{~L} / \mathrm{min}$ of oxygen via non- 
rebreathing reservoir oxygen mask. There were bilateral diffuse crackles on lung examination.

Blood gas evaluation revealed; $\mathrm{pH} 7.34, \mathrm{pCO} 2$ $33.1 \mathrm{~mm} \mathrm{Hg}$, bicarbonate $18.3 \mathrm{mmol} / \mathrm{L}$, lactate $6.3 \mathrm{mmol} / \mathrm{L}(0.4-2.2 \mathrm{mmol} / \mathrm{L})$. We learned that he had been transfused with two units of packed red blood cell, two units of fresh frozen plasma (FFP), and one unit of platelet suspension at the previous hospital. A complete blood count with differential revealed a WBC level of $7,460 / \mathrm{mm}^{3}$, a hemoglobin level of $9.84 \mathrm{~g} / \mathrm{dL}$, a hematocrit level of $29.6 \%$, and a platelet level of $47,000 / \mathrm{mm}^{3}$. There were schistocytes on the peripheral blood smear and the direct antiglobulin test was negative. Prothrombin time was 22.2 seconds, activated partial thromboplastin time was 31.2 seconds, D-dimer level was $22,900 \mu \mathrm{U} / \mathrm{L}(80-500 \mu \mathrm{U} / \mathrm{L})$, and fibrinogen level was $211 \mathrm{mg} / \mathrm{dl}$. The results of other tests were as follows; serum urea $89 \mathrm{mg} / \mathrm{dl}$, creatinine $1.62 \mathrm{mg} / \mathrm{dl}$, uric acid $9.5 \mathrm{mg} / \mathrm{dl}$, albumin $2.68 \mathrm{mg} / \mathrm{dl}$, lactate dehydrogenase $1725 \mathrm{U} / \mathrm{L}$ (120-300 U/L), AST $620 \mathrm{U} / \mathrm{L}(0-32 \mathrm{U} / \mathrm{L})$, ALT $236 \mathrm{IU} / \mathrm{L}(0-32$ $\mathrm{U} / \mathrm{L})$, and C-reactive protein $0.5 \mathrm{mg} / \mathrm{dl}$ (0-0.5 $\mathrm{mg} / \mathrm{dl})$. Serum electrolytes were within normal limits. Serum C3 level was $73 \mathrm{mg} / \mathrm{dl}$ (90-180 $\mathrm{mg} / \mathrm{d})$, C4 level was $7 \mathrm{mg} / \mathrm{dl}(10-40 \mathrm{mg} / \mathrm{dl})$, and haptoglobin level was undetectable $(<7.44$ $\mathrm{mg} / \mathrm{dl}$, normal range; 30-200). Von Willebrand factor-cleaving metalloprotease (ADAMTS 13) $(71 \%)$, vitamin $B 12(417 \mathrm{pg} / \mathrm{ml})$, and homocysteine $(6.55 \mathrm{mmol} / \mathrm{L})$ levels were detected within the normal range. Bilateral mild diffuse opacification was detected on the chest X-ray (Fig. 1A). Abdominal ultrasonography was consistent with bilateral grade 1 renal parenchymal disease.

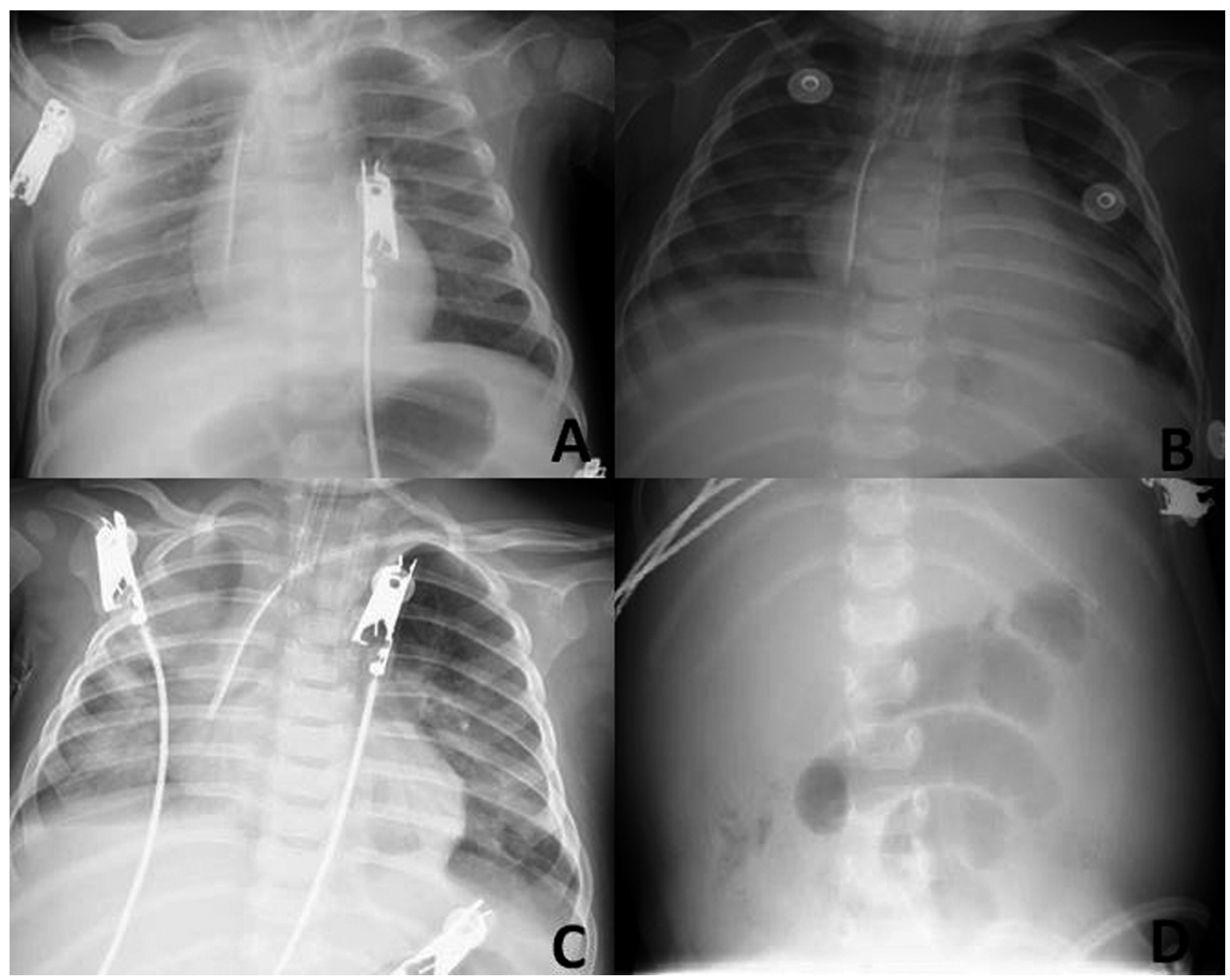

Fig. 1. A) Bilateral mild diffuse opacification on the chest X-ray (1st day), B) Chest X-ray and pulmonary findings (4th day), C) Chest X-ray was consistent with bilateral diffuse opacification after reintubation (4th day), D) Intestine enlargement with no air-fluid level on the abdominal X-ray (10th day). 


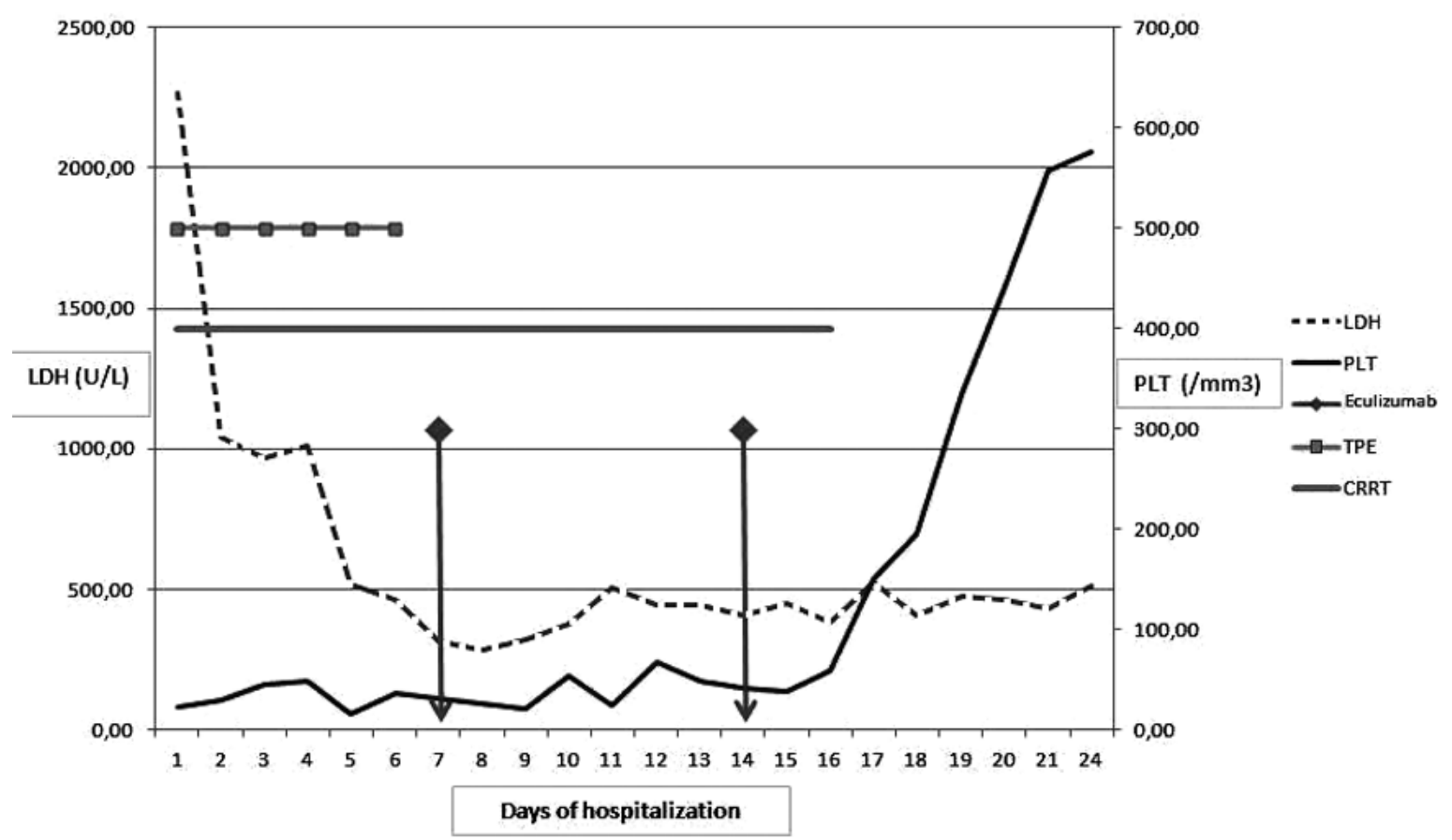

Fig. 2. The course of hematological parameters. CRRT: continuous renal replacement therapy; LDH: lactate dehydrogenase; PLT: platelet; TPE: therapeutic plasma exchange

He was intubated due to respiratory failure and then mechanical ventilation was initiated. After placement of 8-Fr subclavian dialysis catheter, continuous renal replacement therapy (CRRT- venovenous hemodialysis, Prismaflex ${ }^{\circledR}$, HF 20 filter; Gambro Lundia AB, Sweden) at a rate of $2000 \mathrm{ml} / 1.73 \mathrm{~m}^{2} /$ hour and daily TPE (Prismaflex ${ }^{\circledR}$ TP 1000 filter; Gambro Lundie $A B$, Sweden) were started for anuria and preliminary diagnosis of CM-HUS. The first session of TPE was performed with 1.5 volume of FFP and then one volume was used in others. Plasma exchange volume was calculated as follows: $0.065 \mathrm{x} \mathrm{kg} \mathrm{x} \mathrm{(1-} \mathrm{Htc).}$ After seven sessions of TPE, eculizumab therapy was initiated with prophylactic intravenous ampicillin treatment and TPE was stopped. Four days later, edema regressed, chest X-ray and pulmonary findings improved (Figure 1B), so the patient was extubated and noninvasive ventilatory support was initiated. His central venous pressure (CVP) was measured in normal limits $\left(8 \mathrm{~cm} \mathrm{H}_{2} \mathrm{O}\right)$ before extubation. Shortly after extubation, because of the decreasing oxygen saturation and developing respiratory failure he was reintubated. During intubation, pink liquid was seen among the vocal cords moving toward into the mouth which was considered as secondary to pulmonary edema. Chest X-ray was consistent with bilateral diffuse opacification (Fig. 1C). Cardiac contractility function was adequate on repeated echocardiographic examination.

On the tenth day of hospitalization, he had abdominal distention and feeding intolerance. Intestinal sounds were decreased on physical examination without organomegaly. Intestine enlargement with no air-fluid level was seen on the abdominal X-ray (Fig. 1D). Abdomen ultrasonography revealed free liquid in the lower parts of abdomen and minimal perihepaticperisplenic fluid. Additionally, intestines were dilated and immobile. Total parenteral nutrition was started and enteral feeding was stopped. His abdominal findings gradually improved within a week.

On the $16^{\text {th }}$ day, CRRT was stopped since urine output was adequate. Hypertension was controlled with amlodipine and furosemide treatment. On the $17^{\text {th }}$ day, he was extubated. With adequate urine output, no oxygen need in room air, and improved hematological parameters (Fig. 2), he was transferred to pediatric ward on the 23rd day.

Genetic study was performed to confirm the 
definitive molecular diagnosis, treatment and prognosis plan. A new generation of sequencing method involving the complement system and thrombomodulin genes was used. After the genetical study of the CFH, CFI, CFB, CD46, DGKE, CFHR3, CFHR5, CFHR1, C3, C5, and thrombomodulin; nonsense p.Arg1215X terminating mutation was detected in the $\mathrm{CFH}$ gene (Fig. 3). It was decided to continue eculizumab treatment because this mutation causes loss of function in the $\mathrm{CFH}$ protein. Now he is 36 months old; on daily enalapril and eculizumab therapy (every 15 days). His urinary findings are normal and in this period, there has been no CM-HUS recurrence.

Written informed consent was obtained from patient's parent.

\section{Discussion}

Shiga-toxin producing Escherichia coli (STECHUS) is the most common cause of HUS in children. Until recently, all other HUS cases except STEC-HUS were called atypical HUS (aHUS). ${ }^{1,3}$ A new international consensus report was published for classification of HUS terminology. According to this report, it has been recommended that the definition of HUS should be named with the relation of the disease instead of calling it aHUS $^{3}$. The most common cause of HUS except STEC-HUS in childhood is CM-HUS. ${ }^{1-3}$

Complement mediated HUS is a rare disease affecting primarily children and young adults but with high morbidity and mortality rates. It is extremely rare when compared to STEC-HUS and constitutes approximately $5 \%$ of all HUS cases. Although it may occur at any age, it emerges in $70 \%$ of the pediatric patients before the age of 2 years and another $25 \%$ before six months of age. ${ }^{1}$ Despite the appropriate treatment, it causes dialysis requirement, permanent renal damage or death in more than $50 \%$ of the patients. ${ }^{3}$ Most of the disease occurs as a result of mutations in regulatory genes of alternative complement system, but a minor part of it ( 5-6\%) appears as a result of antibodies against to the complement proteins. The most frequent mutation detected in the $\mathrm{CFH}$ gene; and more than 100 mutations related with this gene have been identified. ${ }^{4}$

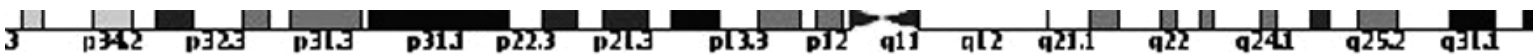

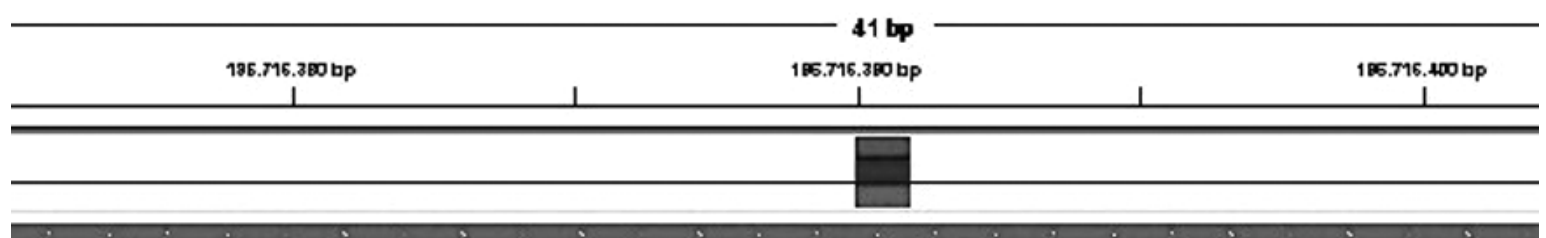

AMPL7154732519

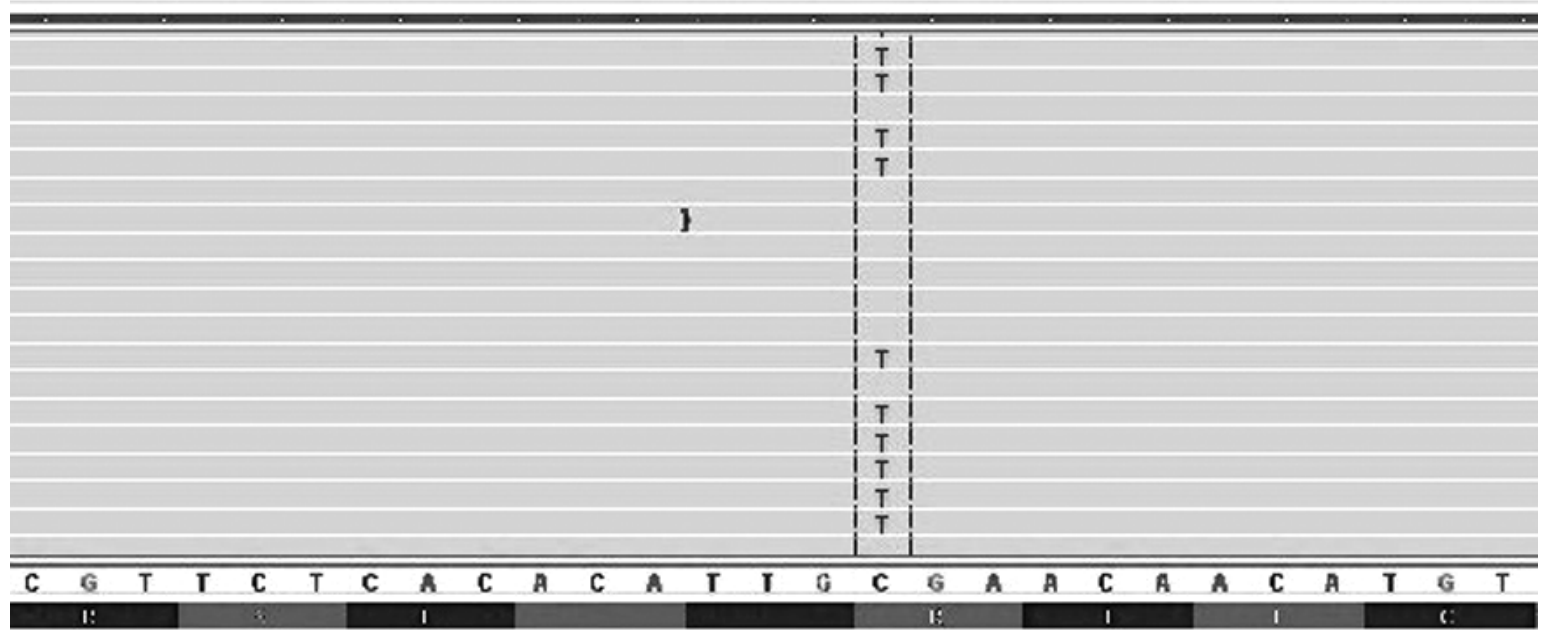

Fig. 3. Nonsense heterozygous p.Arg1215X mutation in the complement factor $\mathrm{H}$ gene. 
Complement $\mathrm{H}$ is the regulator and mainly suppressor protein in alternative complement system. Complement factor $\mathrm{H}$ mutations are classified in two main groups. There is a quantitative deficiency of $\mathrm{CFH}$ in type 1 mutations. In this mutation type, serum $\mathrm{CFH}$ and $\mathrm{C} 3$ levels are low. In type 2 mutations, functional disorder of $\mathrm{CFH}$, serum $\mathrm{CFH}$ and C3 levels are often detected in normal range. ${ }^{1}$ Serum C3 levels are low in $70 \%$ of CM-HUS patients. ${ }^{2}$ In our patient, the heterozygous p.Arg1215X mutation which is considered to be a terminating mutation in the $\mathrm{CFH}$ gene and low C3-C4 levels were detected. Because of the preliminary diagnosis of CM-HUS he was infused twice with FFP in the hospital he came from and later he was treated with TPE with FFP in our PICU. Because of these reasons, $\mathrm{CFH}$ and CFI levels were not analyzed. The p.Arg1215X is extremely rare $\mathrm{CFH}$ mutation in HUS patients and it was reported twice in the available literature.5,6 Fremeaux-Bacchi et al. ${ }^{5}$ genetically analyzed 214 adults and children with CM-HUS in 2013. They have identified a p.Arg1215X mutation but unfortunately they haven't mentioned the clinical course and age of that patient. Phillips et al. ${ }^{6}$ presented a 35 -year-old female patient with the diagnosis of CM-HUS with same mutation in 2016. As distinct from our patient, C3 and C4 levels were normal in that patient and she had no need for dialysis and no extrarenal organ involvement.

Although CM-HUS mostly causes endothelial damage and microvascular thrombosis in renal vessels, it can also affect brain, coronary, intestine, pancreas, liver and lung vessels., ${ }^{2,3}$ Interstitial edema was noted in the effected organs in the autopsy studies of CM-HUS patients. These findings are considered a result of the histamine, $\mathrm{C} 3 \mathrm{a}$, and $\mathrm{C} 5 \mathrm{~b}$ release due to abnormal complement activation. ${ }^{8}$ In addition to TMA, this mechanism could play a role in the increase of intestinal wall thickness, intraabdominal fluid and pulmonary edema observed in our patient. Heart failure and hypervolemia were not considered as a reason of pulmonary edema because of normal echocardiographic findings, vena cava inferior collapsibility index and CVP in our patient.
There are two main treatment choices which are TPE and eculizumab for CM-HUS. If it is technically possible, early and daily TPE are recommended for all suspected CM-HUS patients until the certain diagnosis and genetic studies are finalized.3,7 Eculizumab therapy should be recommended in cases of technical impossibility, unresponsiveness to TPE therapy or with the definitive CM-HUS diagnosis. ${ }^{7} \mathrm{We}$ started to perform daily TPE in the early period and continued until eculizumab was supplied. Renal function and hematological parameters were gradually improved with the TPE and eculizumab (Fig. 2). It was decided to continue the eculizimab therapy after the detection of the p.Arg $1215 \mathrm{X}$ terminating mutation in the CFH gene.

In conclusion, the p.Arg $1215 \mathrm{X}$ terminating mutation in the CFH gene is extremely rare and can cause severe CM-HUS with multiple organ involvement.

\section{REFERENCES}

1. Canpolat N. Hemolytic uremic syndrome. Turk Pediatri Ars 2015; 50: 73-82.

2. Salvadori M, Bertoni E. Update on hemolytic uremic syndrome: Diagnostic and therapeutic recommendations. Worl J Nephrol 2013; 6: 56-76.

3. Loirat C, Fakhouri F, Ariceta G, et al. An international consensus approach to the management of atypical hemolytic uremic syndrome in children. Pediatr Nephrol 2016; 31: 15-39.

4. Rodriguez E, Rallapalli PM, Osborne AJ, Perkins SJ. New functional and structural insights from updated mutational databases for complement factor $\mathrm{H}$, Factor I, membrane cofactor protein and C3. Biosci Rep 2014; 34: e00146.

5. Fremeaux-Bacchi V, Fakhouri F, Garnier A, et al. Genetics and outcome of atypical hemolytic uremic syndrome: A nationwide french series comparing children and adults. Clin J Am Soc Nephrol 2013; 8: 554-562.

6. Phillips EH, Westwood JP, Brocklebank V, et al. The role of ADAMTS-13 activity and complement mutational analysis in differentiating acute thrombotic microangiopathies. J Thromb Haemost 2016; 14: 175185.

7. Greenbaum LA, Fila M, Ardissino G, et al. Eculizumab is a safe and effective treatment in pediatric patients with atypical hemolytic uremic syndrome. Kidney Int 2016; 89: 701-711.

8. Tsai HM. Atypical hemolytic uremic syndrome may present as severe hypertension without hemolysis or thrombocytopenia. Austin J Nephrol Hypertens 2016; 3: 1055. 